\title{
Nonlinear Dynamics Time Series Analysis of Chaotic Current Oscillations in a Semi-insulating GaAs Sample
}

\author{
R.L. da Silva, R.M. Rubinger, A.G. de Oliveira, and G.M. Ribeiro \\ Universidade Federal de Minas Gerais, ICEX, Departamento de Física, \\ CP.702, 30123-970, Belo Horizonte MG, Brazil
}

Received on April , 2001

\begin{abstract}
We have carried out our experiments on a semi-insulating GaAs sample grown by low temperature Molecular Beam Epitaxy. Three parameters were used to fine tune the experiments, namely, temperature, illumination, and applied bias. We have used powerful tools of time series analysis in order to assess the embedding dimension through near false nearest neighbors. We have also measured the maximum Lyapunov exponent and the correlation dimension [1]. The main contribution was in presenting new experimental data and its analysis which presents self-generated chaos.
\end{abstract}

\section{Introduction}

The temporal evolution of a dynamic system with spontaneous oscillations can be measured and recorded in the form of a time series. Our work concerns the experimental study of charge transport in semi-insulating (SI) GaAs samples grown by low temperatures (LT) molecular beam epitaxy (MBE). As a consequence of the low temperature growth, such samples present a high density of As anti-site defects(around $10^{19} \mathrm{~cm}^{-3}$ ) [2]. Under intense bias these samples exhibit spontaneous current oscillations and in certain conditions they present chaotic behavior.

Low frequency oscillations (LFO) are detected in the external current of semiconductor devices in the range of $\mathrm{sub}-\mathrm{Hz}$ to few hundreds $\mathrm{Hz}$. Inside the device they constitute self-organized spatio-temporal structures of the type of electric field domains moving from the cathode to the anode. Their low frequency oscillations are easy to observe and analyze since no sophisticated instrumentation is needed in order to carry out measurements. It is also worth to mention that semiconductor is one of the easily reproducible systems to the study of nonlinear dynamics and deterministic chaotic phenomena. In this way, LFO in semi-insulating (SI) material are a good theme for such studies.

We have studied the $\mathrm{I}(\mathrm{V})$ characteristics of a GaAs sample looking for LFO and route to chaos of the LFO $[3,4,5]$. Semiconductors with negative differential conductivity (NDC) present oscillatory behavior associated with moving high electric field domains that build up spontaneously. Such oscillations occur due to the effect of field enhanced trapping of carriers intro deep traps. The time series were obtained from measurements of the electric current of samples of GaAs. In a context of nonlinear dynamics, our plan was to characterize chaos, correlation dimension and the maximal Lyapunov exponent of these series.

\section{Experimental Details}

To investigate chaotic LFO on the semi-insulating characteristics of GaAs samples we have carried out I(V) measurements in a GaAs epilayer grown by Molecular Beam Epitaxy at low temperature on the top of a LEC GaAs substrate. First, a $1 \mu \mathrm{m}$ thick buffer layer, nonintentionally doped, presenting a p-type characteristic, was grown. After that, the temperature was reduced to $300^{\circ} \mathrm{C}$ and a $2 \mu \mathrm{m}$ thick layer was grown [6]. The low temperature growth is responsible for the high density of the anti-site defect, which was estimated to be around $10^{19} \mathrm{~cm}^{-3}$ [2]. It is well known that the presence of these defects is responsible for the semi-insulating characteristics of the sample [7].

After the sample growth two In contacts were placed $500 \mu \mathrm{m}$ apart. Then the sample was placed in a socket inside cryostat, and lightened with a GaAs LED. The photon flux as measured by replacing the sample with a charged-copled device (CCD) and was found to be $\mathrm{P} \approx 2,2 \times 10^{10} \mathrm{xI}$ (photons $/ \mathrm{cm}^{2} . \mathrm{s}$ ), where $\mathrm{I}$ is the current through the LED in $\mathrm{mA}$. Although the assembly of the measurement circuit is quite simple, it was necessary to take care of random noise, analog to digital conversion error, and the line of $60 \mathrm{~Hz}$. The measurements of the I(V) curve were carried at many temperature and illumination conditions. For the appearance of LFO it is necessary that the sample under investigation presents NDC characteristics. If this condition can be achieved, 
the dc load line of a proper series resistor cuts the I(V) curve in three different points. Two of them define the boundaries (i.e., the amplitude) of the LFO and the other, which stays in the NDC region, generates the instability conditions which gives rise to the oscillations.

As work tool in this characterization of time series, we used a software package (freeware) well-known as project TISEAN [8,9]. This package is distributed freely through a website and it was developed by people with recognized international respectability, being, in that way, reliable.

\section{Results}

The analysis of a LFO measurement was carried out for an applied bias of $35.5 \mathrm{~V}$, at $200 \mathrm{~K}$, and under illumination with $40 \mathrm{~mA}$ through the LED. Figure 1 presents the false neighbor plot. Figure 2 presents the attractor. Figure 3 presents the Lyapunov exponent. Figure 4 shows the correlation dimension. Figure 5 presents the Poincaré map.

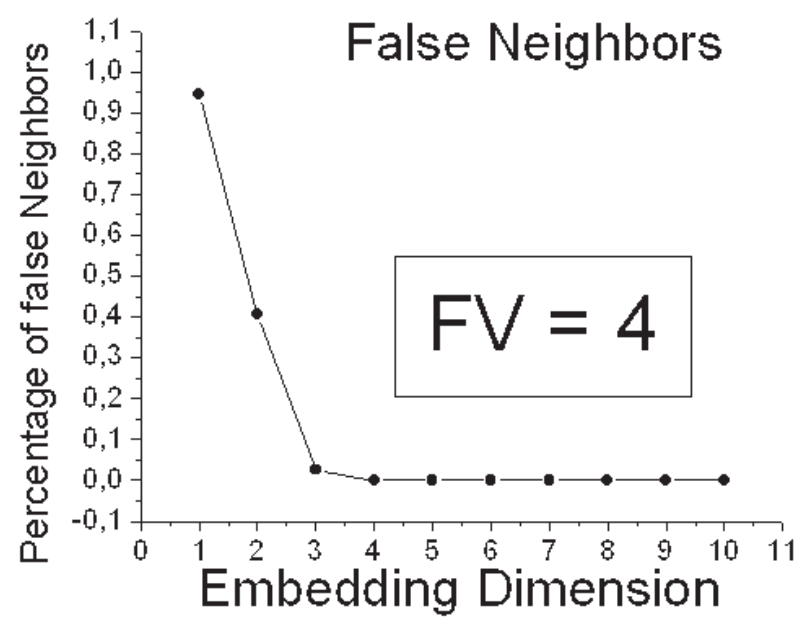

Figure 1. Results of False Neighbors.

Fig. 1 describes the minimum embedding dimension to reconstruct the attractor which is determined by the false neighbors method. For this experimental time series, this method indicates an embedding dimension of 4. Although the suggested embedding dimension is 4, the attractor was represented in a three-dimensional state space in Fig. 2. A reduction in the embedding dimension from 4 to 3 is quite reasonable since the value 4 could be influenced by intrinsic noise of the sample, as has been previously suggested [1].

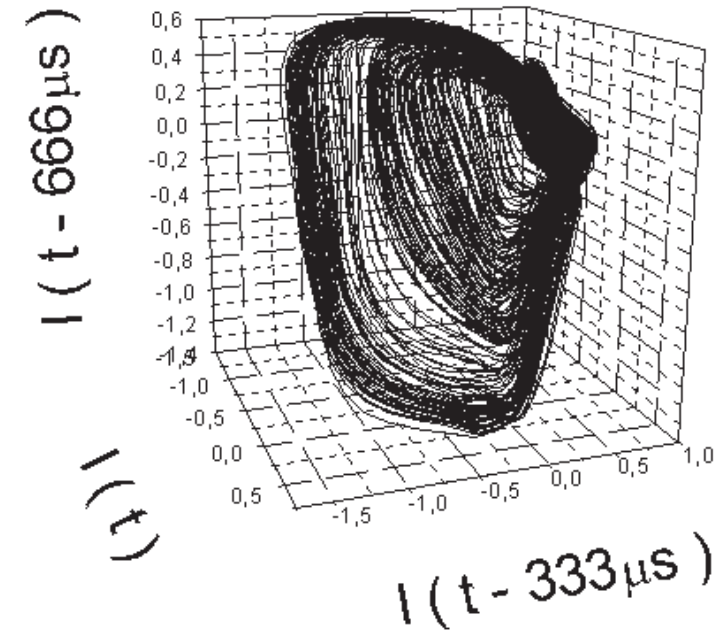

Figure 2. Attractor reconstruction in a three dimensional state space.

Fig. 2 exhibits the attractor reconstructed from the time series in a three-dimensional state space with the three vectors obtained using a delay of 10 steps in the time series.

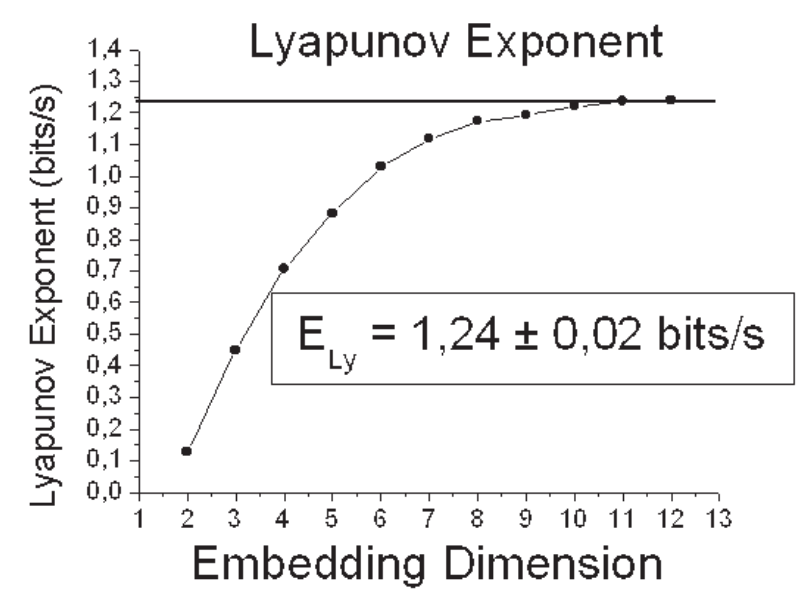

Figure 3. Lyapunov exponent as a function of the embedding dimension.

Fig. 3 shows a value of 1.24 for the Lyapunov exponent. This positive value characterizes the attractor as strange, i.e., chaotic. 


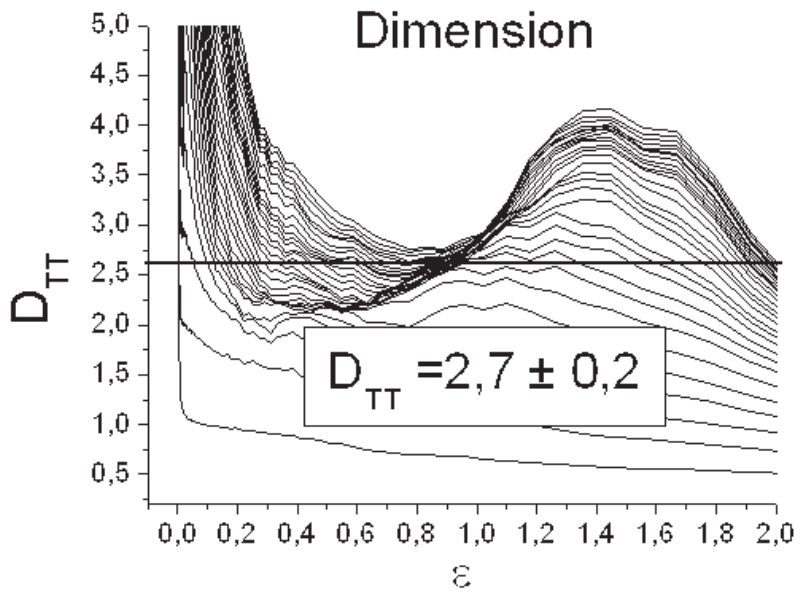

Figure 4. Correlation Dimension estimative.

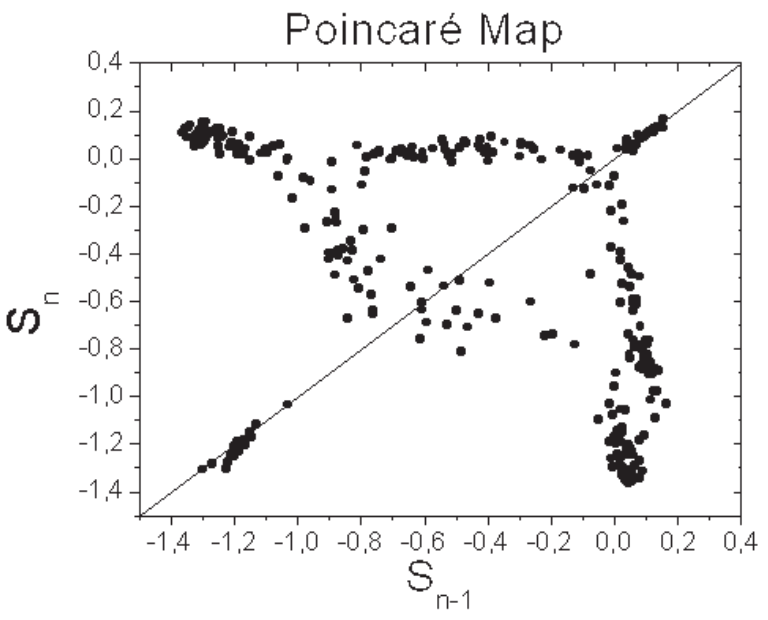

Figure 5. Poincaré Map.

Fig. 4 exhibits the correlation dimension close to 2.7. The Poincaré map presented in Fig. 5 shows two points agglomerate along the straight line $\mathrm{x}=\mathrm{y}$, which indicate two fixed points of the system. Also, it is possible to observe a dispersion of points crossing the $\mathrm{y}=\mathrm{x}$ line, resembling a Poincaré map for a chaotic attractor.

\section{Discussion}

Using the TISEAN package $[8,9]$, it was possible to characterize the experimental time series. The mutual information and the false nearest neighbors provided us with the correct form for reconstructing the attractor[1, 10]. The embedding dimension between 3 and 4 represents a good result, since it is very difficult to obtain experimental noise free data on a highly resistive sample. The obtained value for the embedding dimension suggests a clear attractor structure. The value of $2.7 \pm 0.2$ for the correlation dimension suggests that the experimental system may have three or four freedom degrees. The value of the Lyapunov exponent is also an indication that the system behaves chaotically and, thus, the physical relationship between the system variables and the forces (or fields) is nonlinear.

\section{Summary}

Low Frequency oscillations in a semi-insulating GaAs sample were measured and analyzed with respect to its nonlinear dynamical characteristics. Due to careful measurements it was possible to reconstruct attractors from data with low noise. The values we have found were $1.24 \pm 0.02 \mathrm{bits} / \mathrm{s}$ for the Lyapunov exponent and $2.7 \pm 0.2$ for the correlation dimension.

To our knowledge, there are no measurements of low frequency oscillations in high resistive samples with so favorable signal to noise ratio. The good quality of the measurement results enabled us to obtain accurate values of the Lyapunov exponent and correlation dimension, and also a clear Poincaré map. With such results it is possible to infer the effective generation and recombination processes occurring in this sample and write dynamical equation for simulations of the sample dynamics. We intend pursue further in order a comparison between the experiments and the numerical simulations.

\section{Acknowledgments}

We would like to acknowledge the Brazilian agencies CNPq, FINEP, CAPES, and FAPEMIG for financial support.

\section{References}

[1] H.D.I. Abarbanel, R. Brown, J. J. Sidorowich, and L.Sh. Tsimring, Rev. Mod. Phys. 65, 1331 (1993)

[2] K. Krambrock, M. Linde, and J. M. Spaeth, D.C. Look, D. Bliss, and W. Walukiewicz, Semicond. Sci. Technol. 7, 1037 (1992).

[3] G.N. Maracas and D.A. Johnson, Appl. Phys. Lett. 46, 305 (1985).

[4] E. Scholl, Phys. Rev. B 34, 1395 (1986).

[5] E. Scholl, Phys. Scrip. T29, 152 (1989).

[6] R.M. Rubinger, Braz. Jour. Phys. 29, 797 (1999).

[7] J.S. Blakemore and S. Rahimi, Semiconductors and Semimetals (Academic, New York, 1984), vol.20, 234

[8] R. Hegger, H. Kantz, and T. Schreiber, CHAOS 9, 413 (1999).

[9] TISEAN software http://www.mpipks-dresden.mpg.de/ tisean

[10] H. Kantz and T. Schreiber, Nonlinear Time Series Analysis. Cambridge University Press, Cambridge (1997). 